This item was submitted to Loughborough's Research Repository by the author.

Items in Figshare are protected by copyright, with all rights reserved, unless otherwise indicated.

\title{
Synchronized wireless sensors for aircraft structural health monitoring
}

PLEASE CITE THE PUBLISHED VERSION

https://doi.org/10.1063/5.0033952

PUBLISHER

AIP Publishing

VERSION

AM (Accepted Manuscript)

\section{PUBLISHER STATEMENT}

This article may be downloaded for personal use only. Any other use requires prior permission of the author and AIP Publishing. This article appeared in AIP Conference Proceedings 2309, 020011 (2020); https://doi.org/10.1063/5.0033952 and may be found at https://doi.org/10.1063/5.0033952.

\section{LICENCE}

All Rights Reserved

\section{REPOSITORY RECORD}

Fu, Hailing, Zahra Sharif Khodaei, and MH Ferri Aliabadi. 2020. "Synchronized Wireless Sensors for Aircraft Structural Health Monitoring”. Loughborough University. https://hdl.handle.net/2134/13635047.v1. 


\title{
Synchronized Wireless Sensors for Aircraft Structural Health Monitoring
}

\author{
Hailing Fu, ${ }^{1, \text { a) }}$ Zahra Sharif Khodaei ${ }^{2, b)}$, and M.H. Ferri Aliabadi', c) \\ ${ }^{1}$ Wolfson School of Mechanical, Electrical and Manufacturing Engineering, Loughborough University, LE11 $3 T U$, UK \\ ${ }^{2}$ Structural Integrity and Health Monitoring Group, Department of Aeronautics, Imperial College London, London, SW7 2AZ, UK \\ a) Corresponding author: h.fu@lboro.ac.uk \\ b) Z.sharif-khodaei@imperial.ac.uk \\ c)m.h.aliabadi@imperial.ac.uk
}

\begin{abstract}
This paper presents the design and development of synchronized wireless sensors for monitoring impact events which may occur on composite airframe. Rare, random and transitory impact events, such as bird strike, runway debris or tool drops during maintenance, can introduce barely visible damage to airframes (especially composite structures) which may reduce its load bearing capacity. An innovative wireless sensing skins concept consisting of piezoelectric transducers and wireless transceivers based on Bluetooth Low Energy (BLE) is proposed to monitor the operational condition on-board continuously. An event-based mechanism is adopted for low-power operation while maintaining the sensing performance. The sampling frequency can be set up to $200 \mathrm{kHz}$ while the overall idle state current can be as low as $41 \mu \mathrm{A}$. A synchronization method is proposed and implemented to share common time stamps among multiple wireless sensors. The performance is validated by the results from an oscilloscope. The proposed wireless sensors demonstrate a potential solution for the next generation aircraft structural health monitoring (SHM).
\end{abstract}

\section{INTRODUCTION}

The Internet of Things (IoT) has been an enabling technology that changes the way we live our daily lives. Objects are equipped with smartness with the development of sensing technology, low-power electronics and wireless communication technology [1]. Aeronautical engineering, in this context, is also facing a change in its operation, especially in airborne sensing devices for structural health monitoring. Sensors within a wired network are the current paradigm in evaluating the operation safety, but wired networks introduce significant increase in system weight, wiring difficulty and maintenance complexity. For instance, the electrical wiring in the Airbus A380-800 has a total length of $470 \mathrm{~km}$ and the total weight is around $5700 \mathrm{~kg}$ [2]. This solution not only introduces additional issues to the existing system, but also limits the system capability in obtaining more operational data for comprehensive evaluation of the structural integrity [3].

Compared to wired networks, wireless sensor networks are gaining more and more attentions from both academia and industry. It eliminates the difficulty and complexity of wiring and offers flexibility in sensor deployment. However, there still remains challenges, such as power consumption, signal latency, effective network topology, spectrum requirement and sensing reliability, that need to be addressed before this technology can be adopted in practical large-scale applications in aeronautical engineering $[4,5]$.

There are numerous wireless technologies available, including Wi-Fi, ZigBee, RFID, LoRaWAN, SigFox, NB-IoT and Bluetooth, etc [6]. Most of the current wireless sensors are implemented using the above-mentioned technologies. However, it is worth noting that, for aircraft, there is a recommended spectrum from 4.2 to $4.4 \mathrm{GHz}$ for wireless avionics intro-communication, according to the regulation from the International Telecommunication Union [7]. 
However, due to the availability of off-the-shelf transceivers, majority of wireless sensors for aircraft structural health monitoring in the literature are developed using the spectrum of $2.4 \mathrm{GHz}$ or below.

Different methodologies and prototypes have been developed and presented for aircraft SHM. The progress in this area has been well discussed in several comprehensive review papers [8-10]. Low-power consumption, unobtrusiveness, system scalability, sensing reliability and autonomous operation are considered as the main challenges in wireless sensors for SHM in aeronautical engineering. Joris et al. developed an autonomous wireless sensing system for environmental monitoring using Sigfox [11]. Temperature readings were collected autonomously using these wireless nodes powered by a solar cell. Loubet et al. presented a wirelessly powered sensor node for cyberphysical systems dedicated to SHM applications [12]. A device with a LoRaWAN communication protocol and temperature and humidity sensors was developed and tested. We previous developed two types of wireless sensor nodes for detecting either external impact events or evaluating structural integrity using passive [13] or active sensing [14] methodologies for aircraft SHM.

In this letter, synchronized wireless sensors enabled by BLE and piezoelectric (PZT) sensors are developed to establish smart sensing skins for aircraft on-board structural health monitoring with the features of low power consumption, unobtrusiveness and highly distributed sensing ability. To the authors' knowledge, this integration of BLE and PZT sensors for aircraft SHM in a well-synchronized manner has not been reported previously. The proposed synchronized wireless sensors provide a potential solution towards the realization of the next generation aircraft onboard SHM system.

\section{SYSTEM DESIGN AND OPERATION PRINCIPLE}

\section{Wireless Smart Sensing Skins}

The concept of wireless smart sensing skins for aircraft SHM is illustrated in Figure 1. A Bluetooth mesh network is established to monitor the integrity of critical structures on aircraft, such as stabilizer, wings or fuselage. There are several types of units functioning for different roles in this BLE network, including low power nodes for local sensing, friend nodes for relay and gateways for relay and extending this network to other types for longer-range communication.

The low power nodes act as peripherals and wire-connected with their respective piezoelectric sensors. External events are monitored by the piezoelectric sensors, and the readings are recorded by the local processing module on the low power nodes. Sensing results are sent to the aircraft SHM coordinator or the IoT cloud for further evaluation via friend nodes and relays. The battery-powered low-power nodes do not relay sensing results, and the data are only relayed through mains-powered relay nodes. This allows the low-power nodes to operate over a long term without human intervention for battery replacement or recharging. Energy harvesters can also be adopted for fully autonomous operation for low-power nodes.

BLT-LTE and BLE-WiFi gateways are integrated in this network to fulfil sensing data relay and extend the local network to the Internet. This provides the capability of building a network for inter-connected aircraft. This IoT cloud or the aircraft SHM coordinator on-board will provide a comprehensive evaluation to the aircraft structural health. 


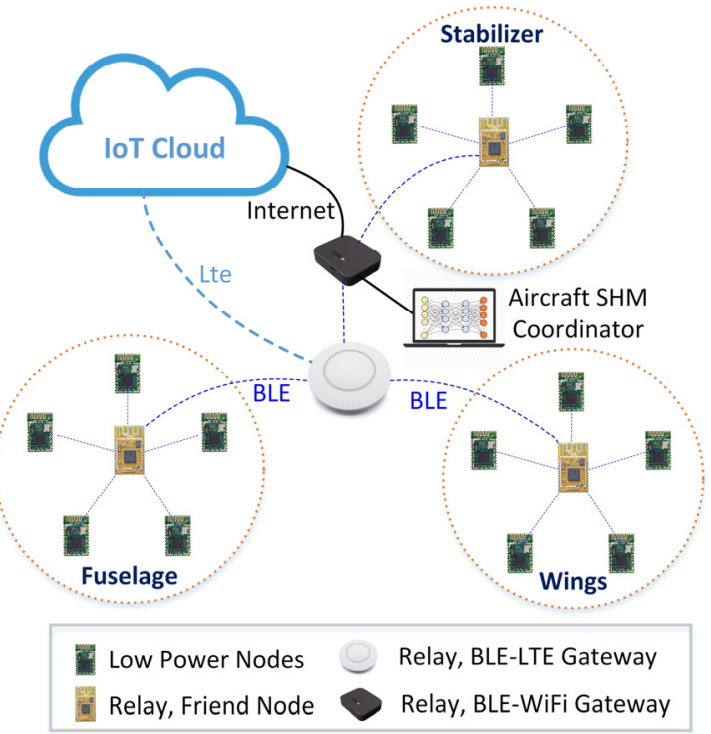

FIGURE 1. Wireless smart sensing skins integrated on critical structures of an aircraft using BLE. Solutions to transmit sensing results from a low power node within a BLE network to the IoT cloud are also provided.

\section{Low Power Node Design}

Each low power node is in charge of a PZT sensor to record the external alarming events when occurred on the host structure. The design of the low-power sensor node is shown in Fig. 2. There are four modules in such a node, including a BLE chip, a PZT sensor mounted on the host structure, a passive rectifier to convert alternating voltages to the direct form suitable to the BLE chip and a comparator module to detect the alarming events determined by pre-set thresholds.

As the impact-induced Lamb waves can oscillate at frequencies as high as several kilohertz, the sensor node sampling frequency should be sufficiently high to maintain the signal quality. However, recording signals at such a high frequency continuously would unavoidably generate plenty of unnecessary data and consume a significant amount of energy from the battery, especially under the situation where external impact events are random, rare and transitory. Therefore, an event-based mechanism is necessary to allow the system to be idle when there is no alarming external event and to be active and record the events only when events occur.

The comparator module in Fig. 2 is designed to fulfil this function. A pre-set threshold is adopted in the comparator as a reference to detect whether the rectified sensing results are higher than the reference. A rising edge will be generated from the comparator output as an indicator that an alarming event has occurred. This indicator will be used as an interrupt for the BLE chip to initiate the recording process enabled by the ADC. As there is time delay for both the comparator and the ADC initiation, a design is required to reduce the time delay to record the beginning part of these transitory events.

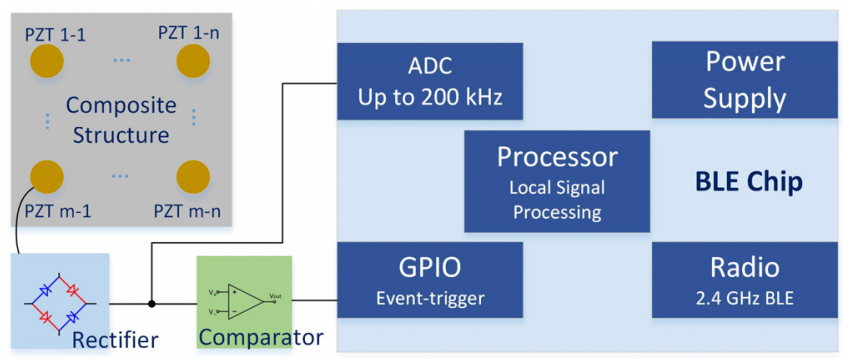

FIGURE 2. Design and configuration of low power sensor nodes for aircraft SHM using PZTs, a BLE chip and an event-based mechanism. 


\section{Hardware Selection and Consideration}

Bluetooth 5 is considered for the BLE chip selection due to its low-power performance and network protocol availability. The chip NRF52840 form Nordic Semiconductors is selected due to the advantages in ADC capability and low-power performance. This chip has a $64 \mathrm{MHz}$ Arm Cortex M4 core with 8 ADC channels sampling at up to $200 \mathrm{kHz}$. A low active current of $0.97 \mu \mathrm{A}$ is realized. The fast wake-up feature is also ideal for this application to initiate the ADC sampling as soon as an external event occurs.

The wake-up trigger is generated by the comparator module. This module also exhibits a response time to generate the trigger signal. This response time should be minimized to capture the full external events. Another factor is the comparator power consumption, as this module is always active to monitor the rectified results. Therefore, a trade-off between response time and power consumption needs to be considered. The comparator TLV3201 from Texas Instruments is selected due to its balance in the response time and consumption.

\section{Timer Synchronization}

As the time of arrival (ToA) for external impact events are critical to determine the impact location, time stamps need to be recorded accurately. However, wireless sensor nodes are distributed widely over a large area and operate with their individual timers. Typically, there are offsets causing inaccurate ToA readings for different sensor nodes. Timer synchronization among all sensor nodes are essential.

A synchronization method is proposed to update the sensor nodes using a shared clock among multiple devices, as shown in Fig. 4. Each node keeps a free-running timer. A master is assigned in the network to transmit synchronization radio packages at a configurable interval. When the other nodes receive this synchronization packet, they can use this value to update their own timer and to synchronize the whole network, as shown in Fig. 3(b).

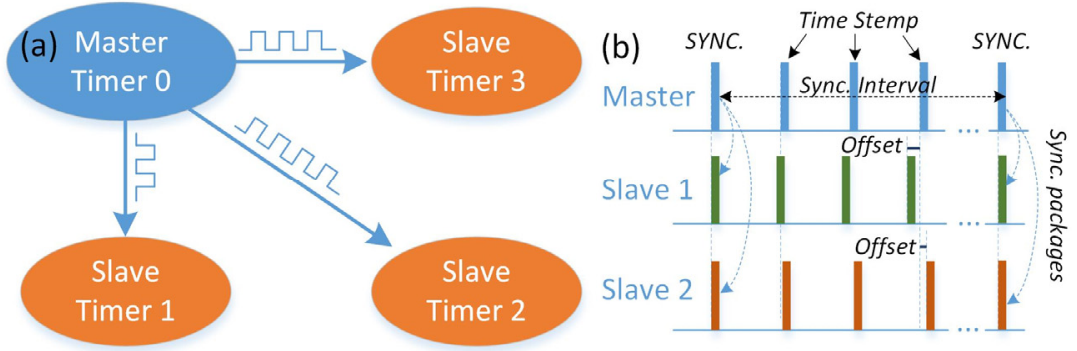

FIGURE 3. Timer synchronization using a shared master clock.

\section{SYSTEM IMPLEMENTATION AND EXPERIMENTAL INVESTIGATION}

The system implementation is illustrated in Fig. 4. The concept of smart skins using wireless sensors are preliminarily investigated on a composite panel with PZTs mounted. Two sensor nodes are adopted, functioning as a friend node and a low power node, respectively. The low power node is connected to a PZT sensor to detect potential external impact events, and the friend node is used as a relay to transfer data wirelessly from the low power node to the aircraft on-board SHM coordinator for impact evaluation.

The rectification and comparison modules are implemented on the low power mode. The detailed design and connection are illustrated in Fig. 5. The PZT sensor is connected to the PZT $+/$ - pins on the rectification module. The

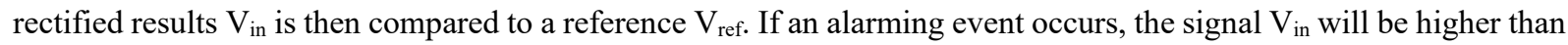
$\mathrm{V}_{\text {ref, }}$, and a trigger signal will be generated at $\mathrm{V}_{\text {out }}$ to initiate the ADC function via a general-purpose input/output pin on the low power node to record the event. Fig. 5(d) shows the generated trigger signal based on a sawtooth input waveform changing between 0 to $3 \mathrm{~V}$ at $2 \mathrm{kHz}$. The reference voltage is set as $0.15 \mathrm{~V}$ by R1 and R2 in Fig. 5(b). A rising/falling edge can be generated when the input signal rises above or falls below the reference, as shown in Fig. 5(d). The response delay is $100 \mathrm{~ns}$, as show in Fig. 5(e). This short delay is ideal for recording the external events 
without losing significant amount of data for the initial part.

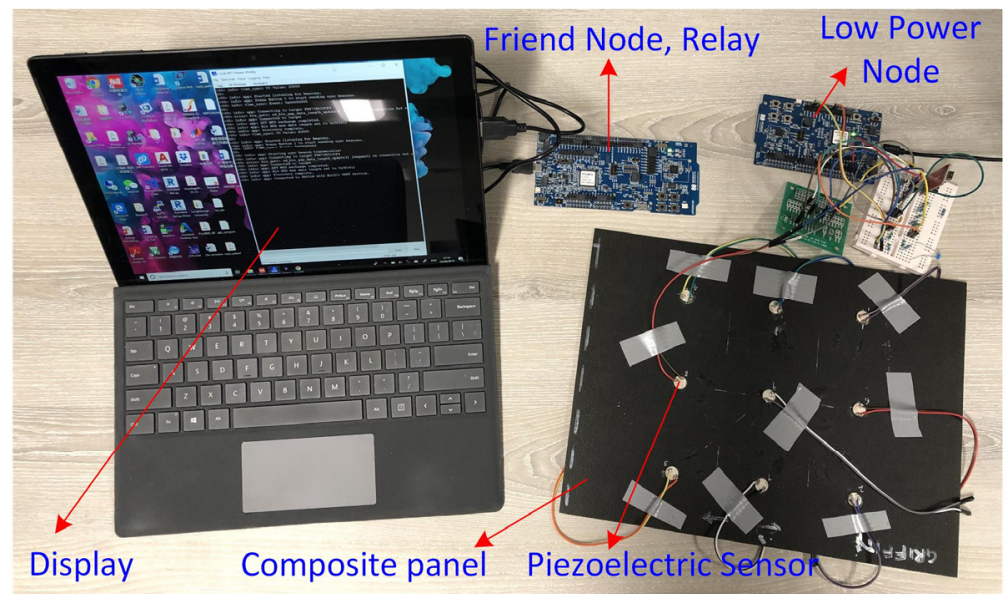

FIGURE 4. Experimental set-up. A low power node is connected with one PZT sensor; the results are wirelessly transmitted to a friend node and eventually to the aircraft SHM coordinator for evaluation.
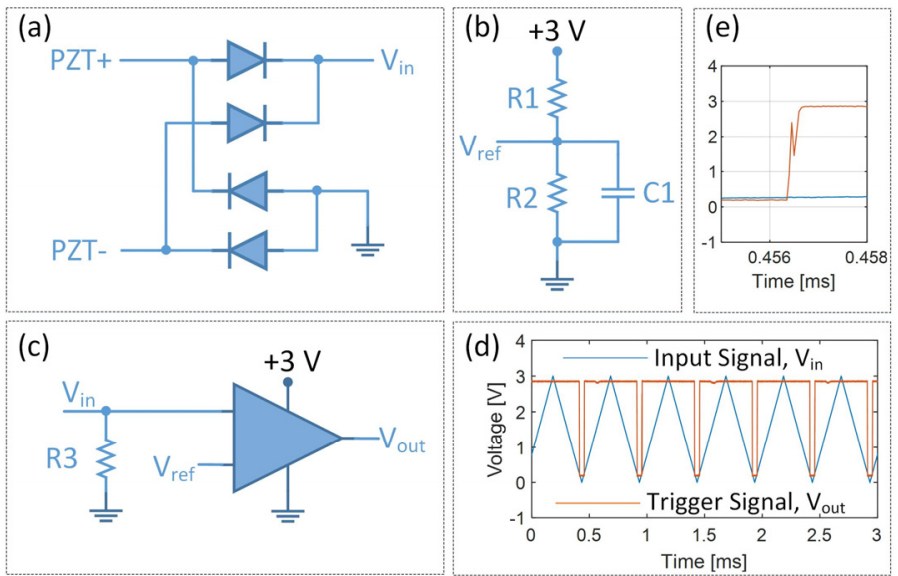

FIGURE 5. Implementation of the rectification circuit (a), reference circuit to provide $\mathrm{V}_{\text {ref }}(\mathrm{b})$, comparator module to generate the trigger signal $\mathrm{V}_{\text {out }}(\mathrm{c})$, the actual trigger signal measured for a sawtooth input (d) and the detailed rising edge when the input is larger than the reference (e).

The sensing performance of the low power node is illustrated in Fig. 6(b) with the comparison to the result measured by an oscilloscope in Fig. 6(a). The sampling frequency of the oscilloscope was set at $10 \mathrm{MHz}$, while the sampling frequency of the low power node was $50 \mathrm{kHz}$. A sufficient accuracy was maintained even though the sampling frequency is much lower. An impact event was introduced at $2 \mathrm{~ms}$; a trigger signal was generated to initiate the ADC function, and the sensing results were recorded by the low power node with marginal signal loss. This event-triggered mechanism allows the ADC and radio functions to be active only when alarming external events occur. An extremely low idle current below $41 \mu \mathrm{A}$ can be obtained when the comparator is active $(40 \mu \mathrm{A})$ and the BLE chip is in sleep $(0.97 \mu \mathrm{A})$. 

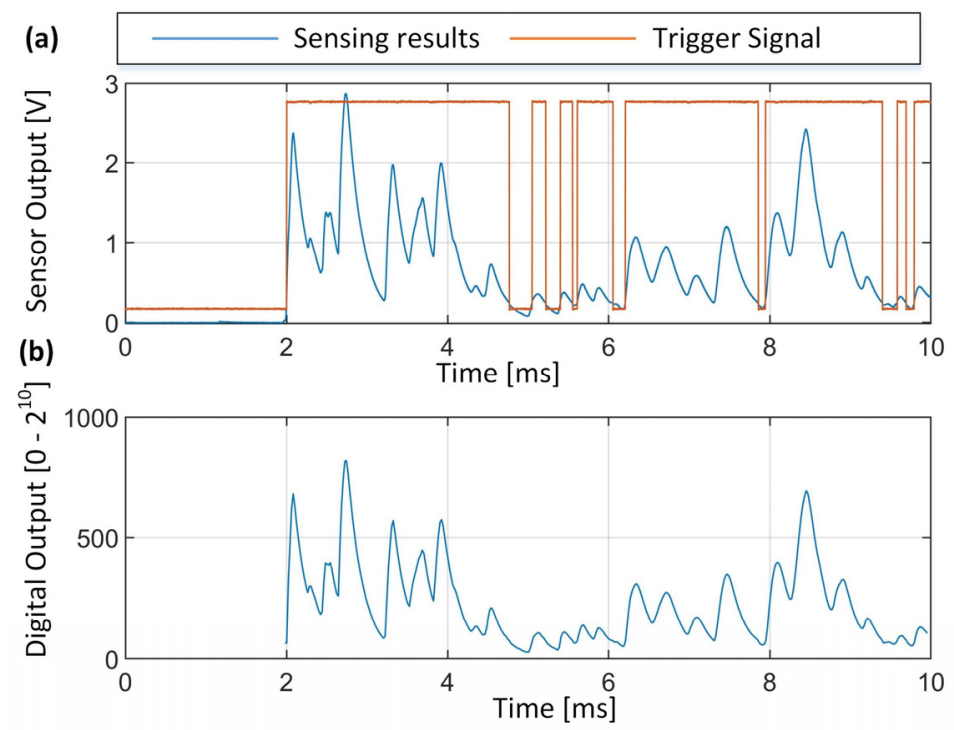

FIGURE 6. Comparison of the sensing results from an oscilloscope and the low power node. (a) Sensing results $V_{\text {in }}$ and generated trigger signal $\mathrm{V}_{\text {out }}$ from the oscilloscope; (b) sensing results from the low power node.

The synchronization method is validate using to wireless BLE nodes, one as the master node and the other as the slave node. Each node keeps a free-running $16 \mathrm{MHz}$ timer with a 16-bit counter. The timer generates a callback event once the timer overflows. Therefore, step curves are generated, as shown in Fig. 7(a) for each node. When the system is unsynchronized, the timers are counting with different time stamps, causing inaccurate sensing results from multiple devices. The master node can transmit synchronization radio packages at a configurable interval. Once receiving the packages, the slave node can update its timer based on the timer information of the master node. The synchronization results are shown in Fig. 7(b). The details of the synchronization result are shown in Fig. 7(c), showing a time-lag of $20 \mathrm{~ns}$. This time lag is marginal compared to the timescale of the considered impact events.
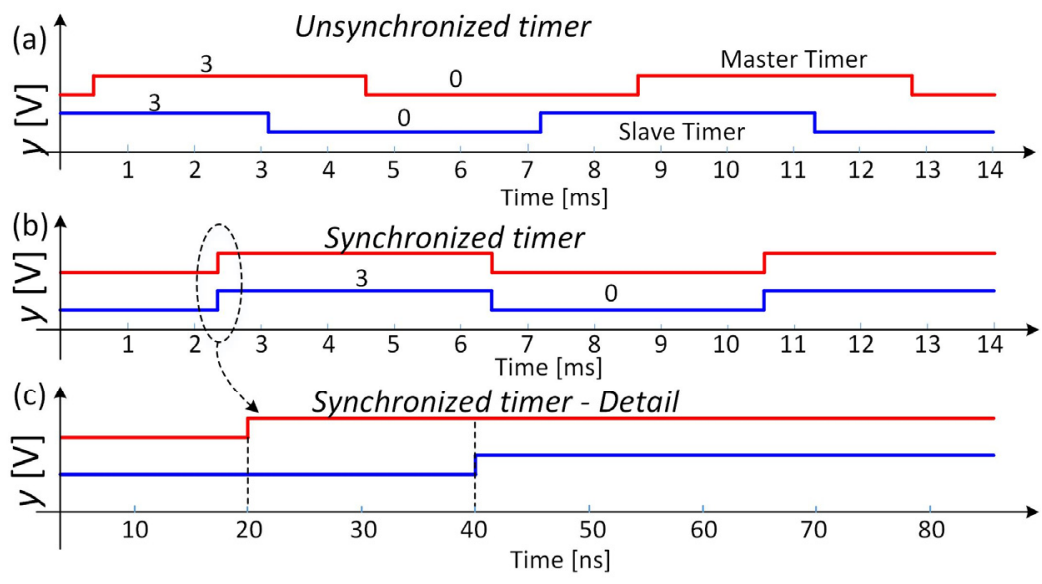

FIGURE 7. Experimental validation of the time synchronization method using one master node and one slave node. (a) Unsynchronized, (b) synchronized and (c) details of the synchronized result.

The multi-channel sensing performance was further examined on a stiffened panel with a stiffener in the middle as shown in Fig. 8(b). The same threshold $0.15 \mathrm{~V}$ was used for different channels and impacts with the same energy level and location (between PZT 1 and 2) were repeated in order to obtain sensing results from 8 channels. Fig. 8(a) shows the output voltages for different channels in time; Fig. 8(b) depicts the RMS voltage for different channels over $5 \mathrm{~ms}$. The location and energy levels can be indicated. 

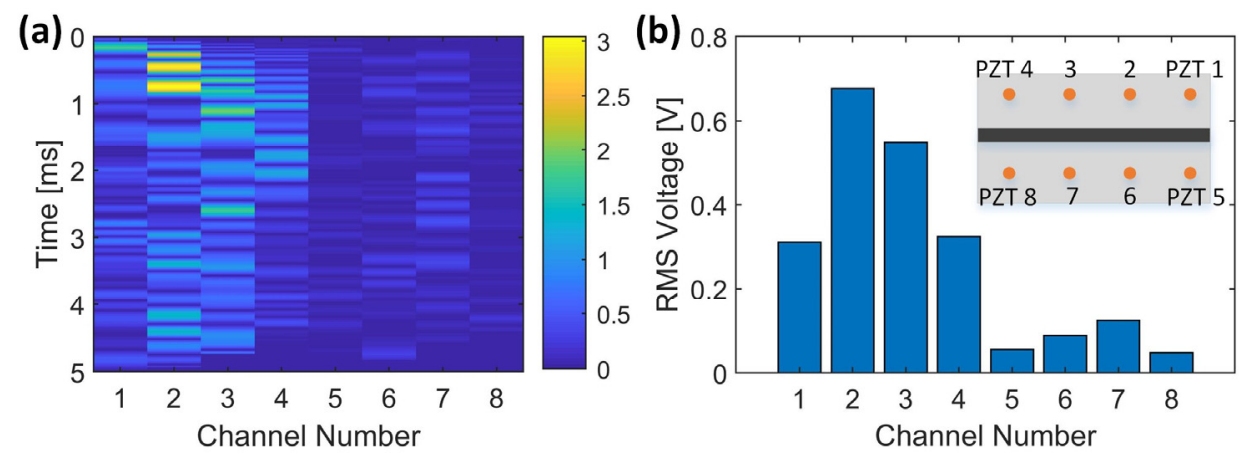

FIGURE 8. Multichannel performance. (a) Sensing results for 8 channels in time. (b) RMS voltage over 5 ms for 8 channels. The data was collected on a stiffened panel as shown in the top-right corner in Fig. 8(b).

\section{CONCLUSIONS}

An aircraft SHM system was developed using wireless sensors and PZTs to detect external impacts on composite airframes. The sensor nodes were synchronized over multiple devices using a shared timer and radio communication. An event-triggered method was realized to reduce power consumption while maintaining the performance. The system was tested on composite panels. An idle current of $41 \mu \mathrm{A}$ was obtained while the sensing sampling frequency can be up to $200 \mathrm{kHz}$ when active. Time synchronization was successfully implemented in two BLE nodes with a marginal time-lag of 20 ns. Multi-channel performance was evaluated on a stiffened panel, and the results demonstrate the capability of the system in identifying external impacts and their locations. Future wok will be on miniaturizing the low power node and evaluating the overall sensing performance on a large scale within a multi-node network. Machine learning will be integrated to achieve a fully autonomous aircraft SHM system.

\section{REFERENCES}

[1] A. Zanella, N. Bui, A. Castellani, L. Vangelista, and M. Zorzi, "Internet of Things for Smart Cities," IEEE Internet of Things Journal, vol. 1, no. 1, pp. 22-32, 2014.

[2] E. W. Rozier, "Data Privacy and Security Challenges for Next-Generation Aircraft: Using Smart-Bridge Technology and Privacy-Preserving Search in Heterogeneous Aircraft Systems," in 17th AIAA Aviation Technology, Integration, and Operations Conference, 2017, p. 3111.

[3] D. Bekas, Z. Sharif-Khodaei, and M. H. Aliabadi, "An innovative diagnostic film for structural health monitoring of metallic and composite structures," Sensors, vol. 18, no. 7, p. 2084, 2018.

[4] D. Krichen, W. Abdallah, and N. Boudriga, "On the design of an embedded wireless sensor network for aircraft vibration monitoring using efficient game theoretic based MAC protocol," Ad Hoc Networks, vol. 61, pp. 1-15, 2017/06/01/ 2017.

[5] L. V. Allmen et al., "Aircraft Strain WSN Powered by Heat Storage Harvesting," IEEE Transactions on Industrial Electronics, vol. 64, no. 9, pp. 7284-7292, 2017.

[6] Y. Mehmood, F. Ahmad, I. Yaqoob, A. Adnane, M. Imran, and S. Guizani, "Internet-of-Things-Based Smart Cities: Recent Advances and Challenges," IEEE Communications Magazine, vol. 55, no. 9, pp. 16-24, 2017.

[7] Available: https://www.itu.int/rec/R-REC-M.2085-0-201509-I/.

[8] X. Qing, W. Li, Y. Wang, and H. Sun, "Piezoelectric Transducer-Based Structural Health Monitoring for Aircraft Applications," Sensors, vol. 19 , no. 3, p. 545, 2019.

[9] M. Abdulkarem, K. Samsudin, F. Z. Rokhani, and M. F. A Rasid, "Wireless sensor network for structural health monitoring: A contemporary review of technologies, challenges, and future direction," Structural Health Monitoring, vol. 0, no. 0, p. 1475921719854528, 2019.

[10] S. Gao, X. Dai, Y. Hang, Y. Guo, and Q. Ji, "Airborne Wireless Sensor Networks for Airplane Monitoring System," Wireless Communications and Mobile Computing, vol. 2018, p. 18, 2018, Art no. 6025825.

[11] L. Joris, F. Dupont, P. Laurent, P. Bellier, S. Stoukatch, and J. Redouté, "An Autonomous Sigfox Wireless Sensor Node for Environmental Monitoring," IEEE Sensors Letters, vol. 3, no. 7, pp. 01-04, 2019.

[12] G. Loubet, A. Takacs, and D. Dragomirescu, "Implementation of a Battery-Free Wireless Sensor for Cyber-Physical Systems Dedicated to Structural Health Monitoring Applications," IEEE Access, vol. 7, pp. 24679-24690, 2019.

[13] H. Fu, Z. S. Khodaei, and M. H. F. Aliabadi, "An Event-Triggered Energy-Efficient Wireless Structural Health Monitoring System for Impact Detection in Composite Airframes," IEEE Internet of Things Journal, pp. 1-1, 2019.

[14] H. Fu, Z. Sharif-Khodaei, and M. H. F. Aliabadi, "An energy-efficient cyber-physical system for wireless on-board aircraft structural health monitoring," Mechanical Systems and Signal Processing, vol. 128, pp. 352-368, 2019/08/01/ 2019. 